\title{
Modulation of the extraordinary optical transmission by surface acoustic waves
}

\author{
D. Gérard*, V. Laude, B. Guizal, S. Benattou, A. Khelif, D. Van Labeke \\ Institut FEMTO-ST, UMR CNRS 6174, Université de Franche-Comté, \\ 32 avenue de l'observatoire, F-25044 Besançon cedex, France
}

\begin{abstract}
The numerical study of periodically nanostructured metallic films exhibiting extraordinary optical transmission (EOT) deposited onto the top of a piezoelectric material is reported. Surface acoustic waves are generated in the piezoelectric substrate and their influence in the transmission spectrum of the EOT structure is studied. It is shown that low frequency acoustic waves can significantly tune the resonance frequency of the EOT structure.
\end{abstract}

\section{INTRODUCTION}

Nanostructured metallic films, such as arrays of subwavelength holes or slits [1, 2], single apertures surrounded by a corrugation [3], or continuous films presenting a periodical texturing of their surface [4], exhibit remarkable optical properties. One of these properties is the so-called "extraordinary optical transmission" (EOT): an array of subwavelength apertures exhibits resonances in its transmission spectrum where the transmission efficiency is significantly higher than one could expect by simply considering the surface occupied by the apertures. The EOT phenomenon has mainly two origins: electromagnetic surface modes at the surface of the metal (surface plasmons) and guided modes inside the apertures (when apertures exist). Another remarkable property of nanostructured metallic films is the presence of high amplitude electromagnetic fields both at their surface and inside the apertures. This is why these structures are promising for a wide range of applications, such as surface enhanced Raman scattering (SERS), angle-independent filtering [5], enhancement of non-linear properties [6], enhancement of single molecule fluorescence [7], polarization of light at the nanoscale [8]... However, for actual technological applications it would be necessary to be able to control the optical properties of the nanostructures by an external signal. Alloptical control (through a third order non-linearity) of the transmission through an array of apertures has been previously reported [9]. However this study evidenced an on/off switching of the EOT, not continuous modulation.

In this paper, we will focus our attention on the control of the EOT by using surface acoustic waves (SAWs) generated in a piezoelectric material. SAWs are usually generated by interdigital transducers (IDTs), consisting of a periodical set of metallic stripes (electrodes). The period of the stripes, which is related to the celerity of the acoustic waves, is typically $500 \mathrm{~nm}$ for a SAW frequency of $3 \mathrm{GHz}$ in a material such as lithium niobate $\left(\mathrm{LiNbO}_{3}\right)$. Such dimensions are well adapted for EOT in the visible and near-infrared region. Therefore, one can imagine

* present adress: Institut Fresnel, Domaine Universitaire de Saint Jérôme, F-13397 Marseille cedex 20, France a device consisting of a metallic grating deposited on a piezoelectric substrate: it would exhibit EOT properties, and its optical transmission would be tunable by using the same metallic grating as a piezoelectric transducer. In the following we will discuss the feasibility of such a device by exploring different geometries with numerical experiments. We will show that indeed, the ideal geometry discussed above (where the EOT structure acts also as an IDT) is not appropriate and will propose a more efficient design. We use a simple numerical approach in two steps. Rather than using some "multi-physics" algorithm, we first estimate the acousto-optic effect with one algorithm, and then enter data from the acoustic code in our electromagnetic solver.

The paper is organized as follows. In Section II we present the EOT structure, without considering any acousto-optic interaction. The structure is a onedimensional metallic grating deposited on the top of a lithium niobate substrate, and it exhibits a high amplitude transmission resonance. Section III is devoted to the study of the acousto-optic interaction in the nanostructured metallic film, in the case where the metallic grating acts as a piezoelectric transducer. In Section IV we present another design, where a low frequency SAW changes globally the refractive index of the substrate. We show that this approach can lead to a strong tunability of the transmission resonance, providing that the acousto-optic effect is high enough. Finally in Section V we summarize our results and draw some perspectives.

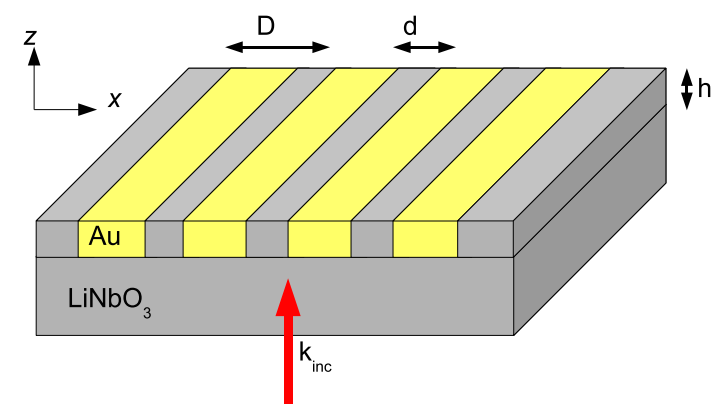

FIG. 1: (Color online) Sketch of the EOT structure. 


\section{EXTRAORDINARY TRANSMISSION THROUGH A 1D METALLIC GRATING ON LITHIUM NIOBATE}

The structure under consideration is schematically drawn on Fig. 1. It consists of a one-dimensional gold grating deposited onto a semi-infinite Y-cut $\mathrm{LiNbO}_{3}$ substrate. The structure is assumed to be infinite in the $y$ direction, and to be periodic in the $x$ direction. The $y$ axis is aligned with the $\mathrm{Z}$ crystallographic axis. The slits of the grating (i.e., the space between each metallic stripe) are filled with $\mathrm{LiNbO}_{3}$. The period of the grating is $D$, the width of the slits is $d$ and the gold film thickness is set to $h$. The dielectric permittivity of gold is taken from tabulated experimental data [10]. The grating is illuminated under normal incidence from the substrate side by a monochromatic, $p$-polarized (electric field perpendicular to the direction of invariance of the slits), plane wave.

The most important assumption we made in the computations is that the substrate is isotropic. Strictly speaking, this assumption is obviously wrong since $\mathrm{LiNbO}_{3}$ is an anisotropic (uniaxial) material. In order to check the role played by the anisotropy of the substrate, we have made numerical simulations of an uniform gold film deposited on a $1 \mu \mathrm{m}$ thick Y-cut $\mathrm{LiNbO}_{3}$ layer, this layer being deposited onto the top of a semi-infinite glass substrate. To compute the reflection efficiency through the layered structure, a T-matrix method has been employed [11]. On Fig. 2 is reported the reflectivity as a function of the angle of incidence, first considering the substrate as an isotropic material (with a refractive index set to the average value between the ordinary index and the extraordinary index) and second by taking into

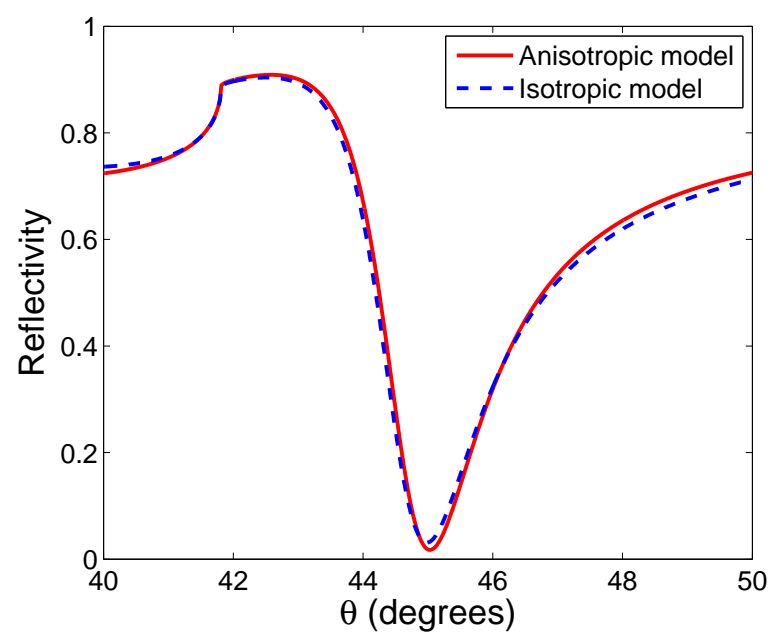

FIG. 2: (Color online) Computed reflectivity of a gold film deposited on a prism, in a attenuated total reflection setup. Solid line: the anisotropic dielectric tensor is taken into account. Dashed line: the substrate is approximated by an isotropic layer.

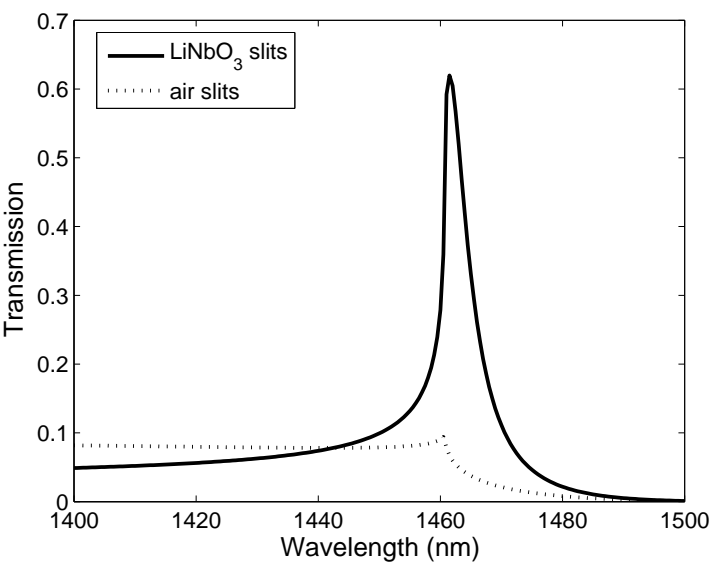

FIG. 3: Transmission spectrum through a grating with $\mathrm{D}=650 \mathrm{~nm}, \mathrm{~d}=100 \mathrm{~nm}$ and $\mathrm{h}=90 \mathrm{~nm}$, when the slits are filled with lithium niobate (solid line) and when the slits are filled with air (dotted line).

account the anisotropic tensor of the dielectric permittivity. In each case a strong dip of reflectivity appears on the spectrum, associated to the excitation of a surface plasmon on the gold/air interface. Comparison between the isotropic and anisotropic cases shows that the anisotropy does not influence much the plasmon resonance. The position of the reflectivity minimum is shifted by less than $0.05^{\circ}$ and the resonance width is the same. It should be stressed that the plasmon resonance of an interface is extremely sensitive to small variations in the refractive indexes of the neighboring media. This is a strong indication that the optical properties of our metallic grating (mostly governed by plasmon resonances and guided modes inside the apertures) will not be affected much by the anisotropy of the substrate. A similar behavior has been observed experimentally by Sun and co-workers [12]. As a consequence, in all the following calculations, the lithium niobate substrate is assumed to be an isotropic and non dispersive material, its dielectric permittivity being set to $\varepsilon=5.05$.

All electromagnetic calculations presented below were made using the Fourier Modal Method (FMM) equiped with the S-matrix algorithm to prevent instabilities [13] and the correct rules of Fourier factorization to improve convergence $[14,15]$. On Fig. 3, solid line, is plotted the zeroth order transmission through a structure with period $D=650 \mathrm{~nm}$, slit width $d=100 \mathrm{~nm}$ and thickness $h=90 \mathrm{~nm}$. A high amplitude transmission resonance is observed, with a reasonably good quality factor. The finesse of the resonance is in stricking contrast with the low quality factor that is generally linked to the high transmittance peaks of slits gratings [2]. Generally speaking, EOT structures presenting a good transmittance (like annular apertures arrays [16]) rely on guided modes, but the drawback is that this high transmittance is associated with relatively large resonance peaks. In contrast, 


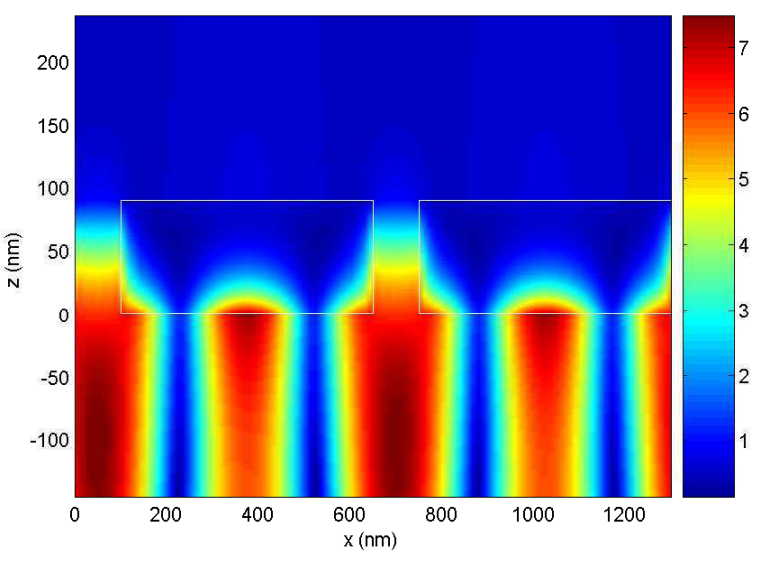

FIG. 4: (Color online) Amplitude of the magnetic field inside the structure and in its near-field at the resonance wavelength. The grating is illuminated from below. The white rectangles shows the boundaries of the metallic grating.

here we obtain a transmission resonance combining good transmission and a quite good quality factor. We attribute this resonance to the coupling between a surface plasmon propagating along the silver $/ \mathrm{LiNbO}_{3}$ interface and an evanescent guided mode inside the slits. This assumption is well supported by the following facts: (i) if the slits are filled with air rather than $\mathrm{LiNbO}_{3}$, then the resonance is destroyed (see Fig. 3, dotted line), because of the impedance mismatch between the surface plasmon and the guided mode at the entrance of the slit. (ii) The position of the resonance is given approximately by the product of the refractive index of the substrate times the period, indicating a surface plasmon excitation at the metal/substrate interface. This property of the resonance will be exploited in Section IV. (iii) The near-field map at resonance (see Fig. 4) evidences a high optical intensity both at the metal surface and inside the slits. This also suggests the presence of both a surface wave (horizontal resonance) and a guided mode (vertical resonance) [17]. The hybrid nature of the resonance is interesting for the applications we are looking for since it implies that the resonance frequency may be controlled either by tuning the guided mode or the surface mode.

\section{ACOUSTO-OPTIC INTERACTION IN A NANOSTRUCTURED METALLIC FILM}

From now on we consider that a SAW (Rayleigh wave) is propagating along the surface of the piezoelectric substrate and is interacting with light incident on the nanostructured metallic film from the lithium niobate substrate side. Three effects are a priori expected : (i) modification of the refractive index of the substrate (and, to a much smaller extent, of the refractive index of the metal) ; (ii) physical deformation of the surface due to the prop- agation of the acoustic wave, leading to the appearance of a "dynamic grating" ; (iii) charge interaction between surface plasmons and the electric wave that propagates in the piezoelectric material along with the SAW. Effect (iii) is difficult to quantify and to the best of our knowledge such an interaction has never been reported. It will be neglected in the following. Effect (ii) (physical deformation of the film) was studied by Sun, Shiokawa and Matsui [12] in the case of an unstructured silver film on $\mathrm{LiNbO}_{3}$. They showed, in an attenuated total reflection setup, the effect of the acoustic dynamic grating on the plasmon resonance. However, this effect is pretty weak since the actual deformation of the surface of the film is less than one nanometer high. Therefore, we will also neglect that kind of interaction and we will focus our attention on the acousto-optic effect, that is to say on the modification of the refractive index of the piezoelectric material (the acousto-optic effect in the metal can also be neglected due to the weak acousto-optic constants of gold).

In the following, we consider that the SAW is generated by the metallic grating itself used as an IDT. As a consequence, the wavelength of the subsequent SAW will be twice the period of the grating (the factor of 2 comes from the interdigitation). The dielectric constant of the substrate (and also of the lithium niobate filling the slits) will be periodically modulated, roughly following the waveform of the SAW. Before running any electromagnetic calculation, the first step is therefore to estimate the acousto-optic variation of the refractive index of the substrate. The acousto-optic modulation of the refractive index can be modeled for a SAW propagating in the $x$ direction by:

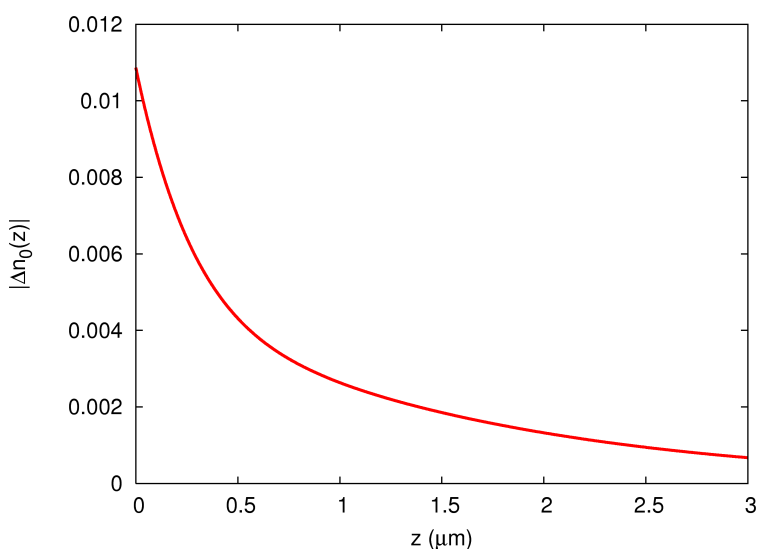

FIG. 5: Modulus of the maximum amplitude of the acoustooptic modulation of the refractive index as a function of the distance to the interface. The SAW is propagating on Ycut lithium niobate along the $\mathrm{Z}$ direction with a velocity of $3490 \mathrm{~m} / \mathrm{s}$ and the frequency is $2.68 \mathrm{GHz}$. The acoustic aperture is set to $30 \mu \mathrm{m}$ and the acoustic power to $1 \mathrm{~W}$. 


$$
\Delta n(x, z)=\Re\left(\Delta n_{0}(z) \exp (i(\omega t-k x))\right)
$$

where $\Delta n_{0}(z)$ is the maximum amplitude of the modulation at height $z$. Inside a SAW resonator, a similar expression holds but the temporal and spatial dependences separate. Principles of the computation of $\Delta n_{0}(z)$ are given in Appendix A. In this calculation, we assume that the metallic stripes do not disturb the SAW propagating on the interface. Fig. 5 shows the modulus of $\Delta n_{0}(z)$ propagating on Y-cut lithium niobate along the $\mathrm{Z}$ direction. The SAW velocity is $3490 \mathrm{~m} / \mathrm{s}$ and the frequency is $2.68 \mathrm{GHz}$ for $D=650 \mathrm{~nm}$. The acoustic aperture is set to $30 \mu \mathrm{m}$ and the acoustic power to $1 \mathrm{~W}$. It is clear from this figure that the maximum modulation is concentrated in the first micrometer below the surface of the substrate. From an electromagnetic point of view, the acousto-optic modulation can be modeled by layering dielectric gratings in the $z$-direction. The value of the acousto-optic modulation inside the slits is set to the same value as the $z=0$ layer of the substrate.

The resulting transmission spectrum is not presented because it is indistinguishable from the undisturbed spectrum of Fig. 3. At first sight, this result could be attributed to the relatively low values of the acoustic perturbation of the refractive index. However, even if $\Delta n_{0}(z)$ is artificially increase by an order of magnitude, the transmission spectrum remains unaffected. Actually, the position of the transmission peak is governed by the spatial average value of the refractive index of the substrate. Since the SAW brings a periodic modulation which is null on average, no effect can be expected on the transmission spectrum. Hence, using the metallic grating exhibiting EOT as an interdigital transducer is an unefficient solution, and other interaction geometries must be looked for.

\section{MODULATION AND TUNABILITY OF THE EXTRAORDINARY TRANSMISSION}

As shown in the previous section, the position of the resonance peak in the transmission spectrum is mainly governed by the average index of the substrate. Consequently, to achieve EOT modulation, it is necessary to modulate globally the refraction index of the substrate. A simple way to do this is to use a low frequency (i.e. high wavelength) SAW. Since an actual nanostructured metallic film for EOT is rarely bigger than a few tens of micrometers, a SAW with a wavelength of a few hundreds of micrometers will be large enough to globally affect the substrate. Keeping these assumptions in mind, we have performed numerical simulations of the transmission spectrum of our nanostructured metallic film (see Section II), for different values of the substrate index. Please note that the value of the refractive index inside the slits is set to the same value than the substrate. As shown on Fig. 6a, the position of resonance shifts with
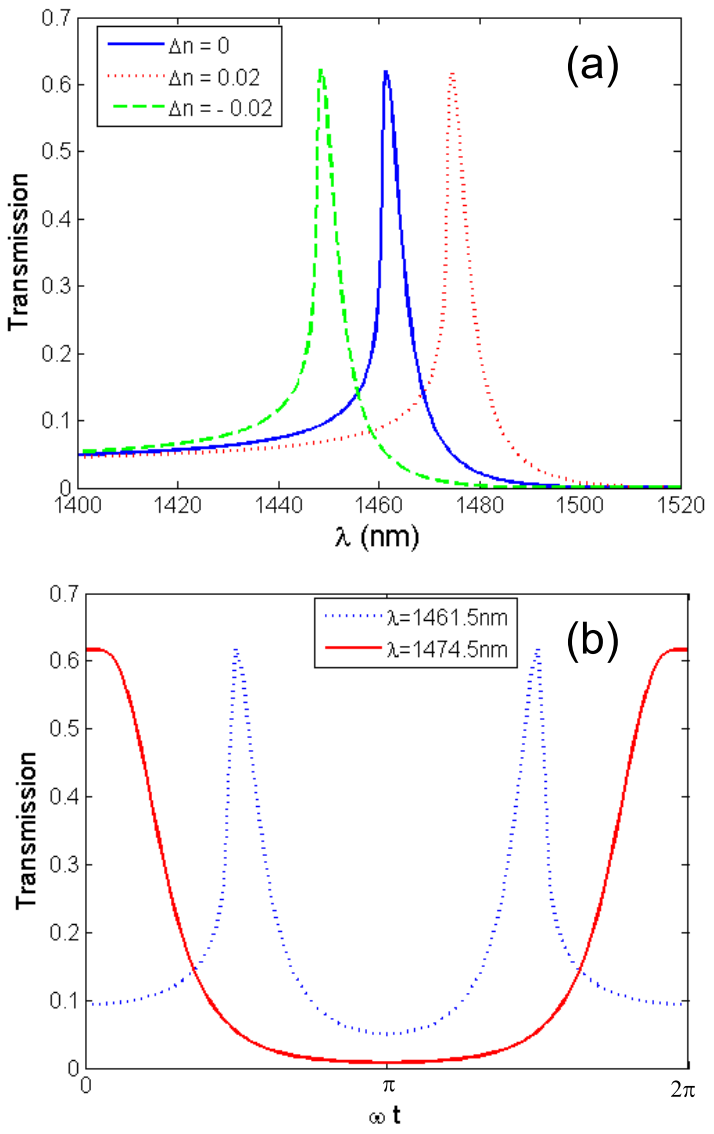

FIG. 6: (Color online) Modulation and tunability of the EOT by a low frequency SAW. (a) Transmission efficiency through the EOT structure for different values of the refractive index of $\mathrm{LiNbO}_{3}$ : no variation (solid line), $\Delta n=0.02$ (dotted line) and $\Delta n=-0.02$ (dashed line). (b) Transmission efficiency vs. time for a maximum modulation $\Delta n_{0}=0.02$ at $\lambda=1461.5 \mathrm{~nm}$ (dotted line) and $\lambda=1447.5 \mathrm{~nm}$ (solid line).

the refractive index. Moreover, the quality factor and amplitude of the peak are not deteriorated. The position of the resonance is roughly given by $n_{s} \times D$, where $n_{s}$ is the refractive index of the substrate. Therefore the position of the transmission maximum follows a linear law, and an acousto-optic variation of the refractive index of $\Delta n=0.0015$ is necessary to shift the resonance by $1 \mathrm{~nm}$. The amplitude of the shift can be further increased for higher values of the grating period, that is to say, for EOT at higher wavelengths. In terms of modulation, Fig. 6a shows the maximum displacement of the resonance, i.e. the spectrum when $\Delta n= \pm \Delta n_{0}$. In order to see the modulation effect, we have plotted on Fig. $6 \mathrm{~b}$ the transmission efficiency at two given wavelengths versus time, taking for the temporal evolution of the acoustooptic modulation $\Delta n=\Delta n_{0} \cos (\omega t)$. It appears that for $\Delta n_{0}=0.02$, a significant modulation of the transmission is observed. For $\lambda=1461.5 \mathrm{~nm}$ (position of the resonance without the acousto-optic modulation), the transmission 
efficiency is modulated from about $61 \%$ at maximum to less than $10 \%$. The rejection of the signal is therefore quite low. The rejection power is improved if the wavelength is set to $\lambda=1474.5 \mathrm{~nm}$ (red solid line on Fig. $6 \mathrm{~b})$ : in this case the transmission drops to about $0.8 \%$ at $\omega t=\pi$.

Now let us focus our attention on the acoustic part of the problem: is it possible to obtain such a $\Delta n$ for low acoustic frequencies in a material like $\mathrm{LiNbO}_{3}$ ? Our calculations show that for classical IDTs, the value of the acousto-optic $\Delta n$ tends to decrease at low frequencies. For instance in $\mathrm{Y}$-cut, Z-propagation lithium niobate at $f=50 \mathrm{MHz}$ (acoustic wavelength $=70 \mu \mathrm{m}$ ), we obtain $\Delta n=2.6 \times 10^{-4}$ for an acoustic power of $1 \mathrm{~W}$ and an acoustic aperture of $1 \mathrm{~mm}$. These values are far from sufficient to obtain tunability of the transmission peak. In order to achieve such an high acousto-optic interaction, it will be necessary to use more efficient acoustic transducers in order to concentrate the acoustic energy. For instance, annular acoustic transducers can be designed to resonantly focus the acoustical power at their center [18]. If an EOT grating were located at the center of such a device it would experience a strong acoustic field, sufficient a achieve optical tunability and modulation with a reasonably good bandwidth.

\section{CONCLUSION}

We have reported a study of the optical properties of an EOT structure deposited onto the top of a piezoelectric material. A simple structure presenting a high amplitude transmission resonance has been proposed. This resonance is linked to the excitation of a surface plasmon along the substrate/metal interface, and its spectral position can be tuned by changing the substrate refractive index. A low frequency SAW could be used to obtain this global change of refractive index. A good tunability is observed, providing that the index variation is high enough. The main difficulty in the actual realization of such structures will be to obtain sufficiently high values of $\Delta n$. Acoustic resonators could be a elegant way to concentrate acoustic power on an EOT device in order to reach an useful tunability range. Such resonators are currently under investigation in our group.

This work has been supported by the Centre National de la Recherche Scientifique and the Région FrancheComté.

\section{APPENDIX A: REFRACTIVE INDEX VARIATION INDUCED BY A RAYLEIGH SURFACE WAVE}

In this appendix, we derive the computation of the refractive index variation induced by a Rayleigh surface wave propagating atop a piezoelectric semi-infinite medium. The formulation we use for expressing Rayleigh surface acoustic waves is based the traditional expansion over partial waves approach, for instance given in Ref. [19]. The displacements inside the piezoelectric medium are expressed as a superposition on partial waves according to

$$
u_{i}=\sum_{j=1}^{4} U_{i j} a_{j} \exp \left(\imath \omega\left(t-s_{1} x_{1}-s_{2}(j) x_{2}\right)\right)
$$

where $x_{1}$ is the coordinate in the propagation direction and $x_{2}$ is the depth coordinate. $\omega$ is the angular frequency, $s_{1}$ is the Rayleigh wave slowness (the inverse of the phase velocity), $U_{i j}$ is a 4 by 4 square matrix gathering the displacements of the partial waves, and $s_{2}(j)$ is the slowness component is the depth of the $j$-th partial wave. The $a_{j}$ are modal amplitudes that are determined from the boundary conditions. Both indices $i$ and $j$ run from 1 to 4 . The fields are assumed to be independent of the $x_{3}$ coordinate. A similar expression is used to express the stress tensor as

$$
T_{k i}=\sum_{j=1}^{4} T_{i j}^{(k)} a_{j} \exp \left(\imath \omega\left(t-s_{1} x_{1}-s_{2}(j) x_{2}\right)\right)
$$

where the index $k$ runs from 1 to 3 . In the domain of existence of Rayleigh surface waves, the $s_{2}(j)$ are complex with a negative imaginary part. It should be noted that they are obtained as the eigenvalues of a generalized eigenvalue problem, the eigenvectors of which also yields the matrices $U_{i j}$ and $T_{i j}^{(k)}$. The free surface or the shorted surface boundary conditions are used here. The Rayleigh wave slowness is then obtained by searching for a zero of the boundary condition determinant as a function of $s_{1}$. This condition determines both $s_{1}$ and the partial waves amplitudes $a_{j}$ up to a multiplicative factor.

The acoustic energy distribution in the depth is treated as follows. The acoustic intensity transported by each partial wave is integrated on a semi-infine surface orthogonal to the propagation direction to give

$$
I(j)=\frac{w\left|a_{j}\right|^{2} P_{1}(j)}{2 \omega \alpha_{2}(j)}
$$

where $w$ is the acoustic aperture, $\alpha_{2}(j)=-\Im\left\{s_{2}(j)\right\}$ and

$$
P_{1}(j)=\Re\left(-\imath \omega \sum_{n=1}^{4} U_{n j}^{*} T_{n j}^{(1)}\right)
$$

is the component of the Poynting vector the $j$-th partial wave directed along the propagation direction. By imposing a given acoustic intensity of the beam, $I$, expressed in Watts, the energetic distribution over the 4 partial waves can be obtained via the normalization relation

$$
I=I(1)+I(2)+I(3)+I(4)
$$

As said above, the partial waves amplitudes $a_{j}$ are only determined up to a multiplicative factor by the boundary 
conditions, i.e. we write $a_{j}=a a_{j}^{\prime}$ where the $a_{j}^{\prime}$ are the known unnormalized values. Inserting Eq. (A4) in Eq. (A5) yields

$$
I=\frac{a^{2} w}{2 \omega} \sum_{j=1}^{4} \frac{P_{1}(j)}{\alpha_{2}(j)}\left|a_{j}^{\prime}\right|^{2}
$$

This equation determines $a$ and then the correctly normalized $a_{j}$ values.

The strain tensor is next derived from Eq. (A1) according to its definition

$$
S_{i j}=\frac{1}{2}\left(\frac{\partial u_{i}}{\partial x_{j}}+\frac{\partial u_{j}}{\partial x_{i}}\right)
$$

This symmetric tensor reads

$$
\begin{aligned}
S_{11}= & -\imath \omega s_{1} U_{1 j} \exp \left(-\imath \omega s_{2}(j) x_{2}\right) a_{j} \\
S_{22}= & -\imath \omega s_{2}(j) U_{2 j} \exp \left(-\imath \omega s_{2}(j) x_{2}\right) a_{j} \\
S_{33}= & 0 \\
S_{23}= & -\imath \omega s_{2}(j) U_{3 j} \exp \left(-\imath \omega s_{2}(j) x_{2}\right) a_{j} \\
S_{13}= & -\imath \omega s_{1} U_{3 j} \exp \left(-\imath \omega s_{2}(j) x_{2}\right) a_{j} \\
S_{12}= & -\imath \omega s_{2}(j) U_{1 j} \exp \left(-\imath \omega s_{2}(j) x_{2}\right) a_{j} \\
& -\imath \omega s_{1} U_{2 j} \exp \left(-\imath \omega s_{2}(j) x_{2}\right) a_{j}
\end{aligned}
$$

The elasto-optic tensor is then used to obtain the variation of the optical impermittivity tensor as

$$
\Delta \eta_{i j}=p_{i j k l} S_{k l}
$$

which translates in the optical dielectric permittivity tensor through

$$
\Delta \epsilon_{i j}=-\epsilon_{i k} \Delta \eta_{k l} \epsilon_{l j}
$$

This last quantity allows one for the computation of the refractive index variation with

$$
\Delta n=\frac{d_{i}^{(1)} \Delta \epsilon_{i j} d_{j}^{(2)}}{2 \sqrt{d_{i}^{(1)} \epsilon_{i j} d_{j}^{(2)}}}
$$

where $d_{i}^{(1)}$ and $d_{i}^{(2)}$ are the components of the unit vectors defining the input and output optical polarization, respectively.
[1] T.W. Ebbesen, H. J. Lezec, H. F. Ghaemi, T. Thio, and P. A. Wolff, Nature (London) 391, 667 (1998).

[2] J.A. Porto, F.J. Garcia-Vidal, and J.B. Pendry, Phys. Rev. Lett. 83, 2845 (1999).

[3] H.J. Lezec, A. Degiron, E. Devaux, R.A. Linke, L. Martin-Moreno, F.J. Garcia-Vidal and T.W. Ebbesen, Science 297, 820 (1998).

[4] D. Gérard, L. Salomon, F. de Fornel and A.V. Zayats, Phys. Rev. B 69, 113405 (2004).

[5] D. Van Labeke, D. Gérard, B. Guizal and F.I. Baida, Opt. Express 14, 11945 (2006).

[6] J.A.H. van Nieuwstadt, M. Standtke, R.H. Harmsen, F.B. Segerink, J.C. Prangma, S. Enoch and L. Kuipers, Phys. Rev. Lett. 97, 146102 (2006).

[7] H. Rigneault, J. Capoulade, J. Dintinger, J. Wenger, N. Bonod, E. Popov and T.W. Ebbesen, Phys. Rev. Lett. 95, 117401 (2005).

[8] F.I. Baida, Y. Poujet, B. Guizal and D. Van Labeke, Opt. Comm. 256, 190 (2005).

[9] I.I. Smolyaninov, A.V. Zayats, A. Stanishevsky, and C.C.
Davis, Phys. Rev. B 66, 205414 (2002).

[10] E.D. Palik (editor), Handbook of optical constants of solids, Academic, New York (1985).

[11] W.C. Chew, Waves and Fields in Inhomogeneous Media, IEEE Press (1995).

[12] X. Sun, S. Shiokawa, and Y. Matsui, J. Appl. Phys. 69, 362 (1991).

[13] L. Li, J. Opt. Soc. Am. A. 20, 655 (2003).

[14] G. Granet and B. Guizal, J. Opt. Soc. Am. A. 13, 1019 (1996).

[15] L. Li, J. Opt. Soc. Am. A. 13, 1870 (1996).

[16] F.I. Baida and D. Van Labeke, Opt. Commun. 209, 17 (2002).

[17] S. Collin, F. Pardo, R. Tessier, and J.-L. Pelouard, J. Opt. A 4, 154 (2002).

[18] C.K. Day and G.G. Koerber, IEEE Trans. Son. Ultrason. SU-19, 461 (1972)

[19] V. Laude, M. Wilm, and S. Ballandras, J. Appl. Phys. 93 (12), 10084 (2003). 